\title{
La psicología educativa a debate en nuestro país: de una encrucijada a algunas alternativas de solución.
}

\section{Educational psychology to debate in our country: a crossroads to any alternative solutions.}

Fecha de recepción: 09-02-2017

Fecha de aceptación: 16-05-2017
José A. León Cascón

Catedrático de Universidad. Departamento de Psicología Básica.

Universidad Autónoma de Madrid

\section{resumen/ahstract:}

Desde sus inicios, la figura del psicólogo educativo en nuestro país y especialmente en lo referente a su ejercicio profesional, ha estado sometido a los dictámenes de las diferentes leyes que han regulado el sistema educativo español basadas en criterios muy diferentes y que abarcan desde la Ley General de Educación hasta la LOE y, la más reciente, la LOMCE. En el momento actual nos encontramos con serios problemas y dilemas con los que se enfrenta nuestra profesión y la formación psicológica especializada. Estos problemas afectan directamente al desajuste entre la formación profesional del psicólogo educativo y su adecuación a las demandas del mercado de trabajo, muchas de ellas inespecíficas o aglutinadas bajo un paraguas de orientación educativa. Por otro, los problemas afectan a diversos modelos de formación, a veces con objetivos opuestos, desarrollados a través de un master en psicología educativa. En este artículo se abordarán dichos problemas junto con algunas de las iniciativas que desde organizaciones avaladas por el COP han tenido lugar en los últimos años y que tratan de responder a los principales desafíos relacionados con la formación, los procesos de acreditación y el rol del psicólogo en el sistema educativo español, así como los criterios de convergencia que se están buscando en Europa entre asociaciones profesionales como la EFPA.

Since its inception, the figure of the educational psychologist in our country, and especially in relation to his professional career, has been subject to the different laws that have governed the Spanish educational system based on very different criteria and ranging from the General Law of education up to the LOE and, most recently, the LOMCE. At the present time, we have serious problems and dilemmas facing our profession and specialized psychological training. These problems directly affect the mismatch between the vocational training of the educational psychologist and its adaptation to the demands of the labor market, many of them non-specific or agglutinated under one umbrella of educational guidance. On the other hand, the problems affect various models of training, sometimes with opposing objectives developed through a master's degree in educational psychology. This article will address these problems together with some of the initiatives that organizations endorsed by the COP have taken place in recent years and trying to respond to the major challenges related to training, accreditation processes, and the role of the psychologist in the Spanish educational system as well as the convergence criteria they are looking for in Europe among professional associations such as the EFPA.

\section{palabras clave/keywords:}

Psicología de la educación, formación especializada, rol del psicólogo escolar, intervención psicoeducativa, acreditación profesional

Educational psychologist, educational guidance, master in educational psychology, europsy. 


\section{dossier}

El artículo introductorio que da sentido a este monográfico sitúa, creo que correctamente, los problemas más importantes que afectan al psicólogo educativo tanto en lo profesional como en lo académico. Básicamente, los problemas del psicólogo educativo en España afectan a su ubicación académica y laboral, lo que conlleva a una indefinición de su propia identidad, referentes a funciones y actividades, a sus perspectivas laborales actuales y de futuro, a su especialización a través de su formación posgrado, a su movilidad laboral, a la continuidad de su formación u homologación tanto dentro del entorno nacional como supranacional... A estos problemas de ubicación e indefinición generalizada, los autores también señalan el de la falta de cohesión. Aunque disponemos de instancias donde crear consensos tanto sobre lo que nos une como sobre lo que nos separa, no se establecen los cauces necesarios para establecer una mesa de diálogo entre los académicos (Conferencia de Decanos, Dptos. e Instituciones académicas como las Facultades de Psicología o las Juntas de Facultad) y los Colegios de Psicólogos ( hay 22, además del Consejo General de Colegios Oficiales de Psicólogos) para tratar y consensuar temas tan cruciales como la formación de postgrado, sobre como regular la formación del psicólogo educativo, sobre la búsqueda de soluciones y alternativas a la encrucijada en la que estamos viviendo.

Lo que no comparto del todo con el artículo introductorio de Emilio Sánchez y Elena Martín es que la causa germinal de esta encrucijada se deba a la inclusión de la Psicología en la rama de conocimientos de las Ciencias de la Salud y de la creación de un master de psicología sanitaria. En mi opinión, la situación actual de la Psicología Educativa viene de la propia división interna de los psicólogos educativos que ya existía mucho antes del 2006, y que sigue aún vigente, tanto conceptual como laboral, y en muchos casos provocada desde los mismos Departamentos de Psicología Educativa y de la Educación, y desde la imposición vivida de una Psicopedagogía que muchos psicólogos educativos no querían, y que veinte años después deja sus corolarios en un reduccionismo laboral y en una formación postgrado demasiado ajena a la psicología educativa propiamente dicha.

Antes de entrar en el desarrollo de estas cuestiones me gustaría señalar que la psicología en España ha entrado en un período de "eclosión". En los últimos años el número de estudiantes que se matriculan cada curso académico en las Facultades de Psicología crece sin parar. Cada año nuevos grados de Psicología aparecen por el territorio nacional. El curso pasado se creó el grado de psicología en la Universidad de Extremadura, con una nota de corte de 10,5. Antes, en la Universidad Rey Juan Carlos. Dada la enorme demanda que está suscitando el grado de psicología que aquellas Facultades de Educación donde antes se impartía el grado de psicopedagogía se está considerando su solicitud como nuevo grado como vía de solución. Por ofrecer algunos datos de este desmedido crecimiento de la psicología en España es que ya supera a Derecho en $n^{\circ}$ de estudiantes, situándose como el grado más solicitado. Esto es algo que sólo ocurre en nuestro país y, en menor grado, en Portugal. Baste señalar aquí que la UNED ha admitido en el curso 2015-16 a más de 37.000 alumnos en el grado de psicología, con asignaturas de primero que alcanzan los 15.000 estudiantes. Y se espera una cifra superior para el curso siguiente. Esto supone más del $40 \%$ de todo el alumnado en España. 
Este fenómeno de expansión de la psicología debería llevarnos a una reflexión general sobre la pertinencia de limitar o no el $\mathrm{n}^{\circ}$ de matrículas en cada universidad, por un lado, y la de ofrecer más salidas profesionales o de formación especializada, por otro. Aunque siempre ha sido la clínica la orientación académica y profesional preferida para la mayoría de los estudiantes de psicología, hoy en día la proporción preferida de los estudiantes se ha incrementado aún más. Es muy posible que parte de esta preferencia provenga de la rama de conocimientos de las Ciencias de la Salud y la creación del master de Psicología General Sanitaria, pero no en detrimento de otras especializaciones. Más bien ocurre al contrario, dado el incremento de alumnos, también se observa un incremento muy significativo en otras especializaciones como la psicología del deporte, la psicología jurídica, psicología del trabajo y de las organizaciones, todas ya por delante de la psicología educativa. Todas ellas ofrecen cada vez más ofertas de posgrado y salidas profesionales. Pero en el caso de la psicología educativa sigue persistiendo un problema de división interna y, si quiere, de inmovilismo que persiste en el tiempo. Trataré en las líneas siguientes argumentar mi opinión, junto a algunas propuestas o alternativas por podría salirse de esta encrucijada.

\section{1.- Sobre la figura del psicólogo educativo en la situación actual}

Desde sus inicios en los años 70, la figura del psicólogo educativo en nuestro país y especialmente en lo referente a su ejercicio profesional, ha estado sometido a los dictámenes de las diferentes leyes que han regulado el sistema educativo español basadas en criterios muy diferentes y que abarcan desde la Ley General de Educación hasta la LOE y, la más reciente, la LOMCE. En este recorrido se ha ido modificando el rol del psicólogo educativo y sus contextos de intervención propugnándose desde un modelo marcadamente médico o centrado en aplicaciones de pruebas y medidas de educación especial, a modelos basados en el asesoramiento y en la intervención sistémica, ecológica o colaborativa. En el momento actual nos encontramos con serios problemas y dilemas con los que se enfrenta nuestra profesión y la formación psicológica especializada. Estos problemas afectan directamente al desajuste entre la formación profesional del psicólogo educativo y su adecuación a las demandas del mercado de trabajo, muchas de ellas inespecíficas o aglutinadas bajo un paraguas de orientación educativa (véase para una revisión Méndez, 2011). Por otro, los problemas afectan a diversos modelos de formación, a veces con objetivos opuestos, desarrollados a través de un master de Psicología Educativa.

En estos últimos años se venido produciendo una desprofesionalización progresiva del estatus profesional del psicólogo educativo diseñado por la Administración Educativa en las sucesivas leyes educativas. En su lugar se ha ido aplicando de manera progresiva un modelo de orientación escolar generalizado (psico) pedagógico en contextos educativos y en donde van desapareciendo las competencias psicológicas (e.g., por competencias didácticas). Recomiendo la lectura de la última ley de educación, la LOMCE, donde podrá observarse que no aparecen ninguna competencia psicológica y en cambio se retoman las competencias didácticas. A esto hay que añadir que viene produciendo una erosión constante y mantenida del estatus profesional producido por la Psicopedagogía (1993-2013) que, al integrarse con la misma salida profesional lleva a la confusión de términos como "orientador", "psi-

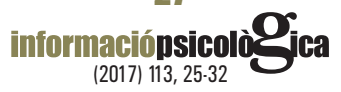


copedagogo", "asesor psicopedagógico", "consultor educativo"..., lo que interfiere en las funciones y atribuciones específicas asignadas anteriormente al psicólogo educativo propiamente dicho. Esta confusión se agranda más aún con otros profesionales y docentes que concurren a las plazas de "orientador". Muy probablemente a esta confusión profesional de la Psicopedagogía y de la orientación se viene manteniendo desde hace más de veinte años una desconexión entre los ámbitos académicos (formación e investigación) y profesional (laboral) y COPs. En muchos casos esta desconexión está alimentada por los propios representantes de ambas instituciones. Frecuentemente oigo comentarios desde las instituciones académicas que sitúan una crítica generalizada hacia los representantes de las instituciones colegiadas como que "siempre" son las mismas personas las que "controlan" los mismos puestos. Curiosamente, escucho estos mismos comentarios generalizados y críticos desde los representantes de las instituciones colegiadas hacia las instituciones académicas de que "siempre" están los mismos catedráticos y en los mismos puestos (Director de Dpto., Decano,...). Una clara evidencia de esa desconexión sigue manteniéndose en el tiempo y que en buena parte se debe a esas divisiones internas y opuestas referente a los defensores de la Psicopedagogía (más mayoritaria en el ámbito académico) y a sus detractores (más representativa en los COPs).

\section{2.- Sobre la formación posgrado del psicólogo educativo.}

Estas divisiones internas se manifiestan aún más cuando se refiere a la formación posgrado y en lo relativo al master de Psicología Educativa. Más específicamente, existen dos tipos de master de Psicología Educativa que pueden cursarse en las universidades españolas. Uno de ellos es el master Psicología Educativa dirigido exclusivamente para los alumnos de grado en psicología. Esto es, se trata de un máster diseñado para los alumnos de psicología (véase a este respecto, Pérez Solís, 2011). Este master está ajustado a contenidos y criterios homologables con otros cursos de posgrados o másteres que se imparten en Europa (León, 2011) Claros ejemplos de donde se imparte este master son la UNED, la UCM, o la Universidad de Murcia. Un segundo master de Psicología Educativa (quizás sería más propio llamarle master de Orientación Educativa) va encaminado a una formación posgrado más general que abarca, además de estudiantes de grado de psicología, a psicopedagogos y a cualquier otro graduado que quiera formase para orientador. Las universidades públicas catalanas, la universidad Autónoma de Madrid o la universidad de La Laguna son algunas de las universidades donde se imparte este posgrado.

Este master así concebido se solapa con el master de formación del profesorado y es criticado por una buena parte de académicos y profesionales de la psicología educativa por su intrusismo profesional. Pero también responde a las propias tensiones internas de los Departamentos de Psicología Evolutiva y de la Educación. Como es sabido estos Departamentos son inter facultativos, pues forman parte de la Facultad de Educación y de la Facultad de Psicología. En muchos casos, como ocurre en las universidades públicas madrileñas, se ha producido un cisma entre los componentes de estas dos facultades, tomando como solución la creación de dos másteres: uno de Formación del Profesorado y liderado por los integrantes de la Facultad de Educación y otro master, Psicología Educativa (Master de 
Orientación Educativa), impartido por los integrantes del Dpto. de Psicología evolutiva y de la educación adscritos a las facultades de psicología, y dirigido al mismo tipo de alumnado, profesores en formación. De hecho en este master de psicología educativa pueda homologarse hasta 60 créditos (de los 90 realizados) con el de Formación del Profesorado. Con este nuevo paso se sigue acentuando las diferencias y divisiones internas entre los psicólogos educativos.

En esta concepciones y diferencias internas también afecta a los dilemas éticos y deontológicos (dificultades derivadas de los procesos de estigmatización, de la confidencialidad y secreto profesional, consentimiento para la intervención y la reivindicación del perfil del psicólogo educativo). El uso de pruebas específicas para psicólogos y que puedan utilizadas por personas poco especializadas. Estos problemas han persistido e incluso algunos de ellos se han incrementado por los sucesivos cambios de legislación, a silencios e indeterminaciones de la ley respecto al perfil del psicólogo en general y del educativo en particular (Méndez , 2011).

En resumen, la psicología educativa se encuentra hoy en día en una difícil encrucijada debido en buena parte en una división interna, tanto académica como profesional, con una disparidad de criterios, a veces opuestos, en la formación de una enseñanza especializada (Máster de Psicología Educativa), en una escasa colegiación de los psicólogos educativos en asociaciones profesionales, lo que puede implicar poca identidad y concientización, poco compromiso profesional, y una desconexión académico-profesional muy alejada de la tradición anglosajona, lo que hace de esta área de la psicología un colectivo débil y vulnerable. Esta situación nos exige una búsqueda urgente de posibles soluciones antes de que la propia psicología educativa siga desvaneciéndose y perdiendo peso dentro de otras áreas de la psicología.

\section{3.- Sobre las tres preguntas planteadas en el artículo introductorio.}

Me posicionare respecto a estas tres cuestiones respondiendo en orden inverso a como se plantean.

Sobre la Orientación Educativa. La tercera pregunta planteada en el artículo introductorio afecta a la única actividad que sí está regulada, al menos, por ahora: la Orientación Educativa. Si bien y desde el punto de vista de los alumnos (de los que cursan el master más centrado en la orientación) podría considerarse imagen de la psicología en el mundo educativo, no estoy de acuerdo en que lo sea desde el punto de vista profesional, ni tampoco considero que sea la imagen más representativa con la que se represente en Europa. Más bien es un avis sui generis de la psicología educativa de nuestro país. Hay que destacar, sin embargo, que hoy por hoy es la salida profesional regulada de la psicología educativa y que, aunque compartida, cuenta con buenos profesionales de la psicología a los que les entusiasma su trabajo y se sienten muy a gusto con su desempeño. En este caso mantendría esta especialización generalizada, pero denominando al master de Orientación Educativa y al que siguieran accediendo otros futuros profesionales. Se trataría entonces de mejorar y profundizar en la especialización y profesión del orientador educativo. El problema sigue siendo un reduccionismo laboral demasiado estrecho para el psicólogo educativo e inviable para la

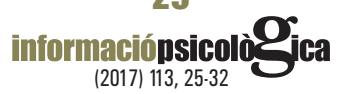


eclosión de la psicología aludida en los párrafos anteriores. Por ello, más que defender una delimitación de actividades profesionales regulables en proceso de diferenciación y exclusivización de los psicólogos educativos en un máster de Orientación Educativa, consideraría como mucho más urgente y necesaria la búsqueda de otras salidas que no tengan que ver necesariamente con la docencia y la orientación.

Sobre la figura del psicólogo educativo no docente. Al igual que ya ha sucedido en otras muchas disciplinas de la ciencia como es la medicina, el cúmulo de conocimientos, cada vez mayor y más difícil de abarcar, hace que, junto a una mayor demanda social, va dando lugar a un mayor número de especialidades (e.g., pediatría, obstetricia, oftalmología, cardiología, alergología, médico de familia,...). La situación de la psicología actual no dista mucho de otras disciplinas y por ello, seguirá ampliándose el número de especialidades. La psicología educativa no es una excepción. Así que no es una cuestión de "urgencias" como se señala en la segunda pregunta, sino un problema de "necesidad" y de "realidad palpable".

El día 21 -9-2010 hubo un atisbo de esperanza al producirse un esperado acuerdo sobre la inserción del psicólogo educativo en el sistema de educación español no universitario. Se respondía así a una demanda educativa, social y profesional que ya venía siendo expresada por multitud de especialistas en el área desde hace bastante tiempo Los miembros firmantes fueron CDPUE (Conferencia de Decanos de Psicología de las Universidades Españolas), el Consejo General de Colegios Oficiales de Psicólogos, la FEDADi (Federación de Asociaciones de Directivos de Centros Educativos Públicos), la CEAPA (Confederación Española de Asociaciones de Madres y Padres de Alumnos), la CONCAPA (Confederación Católica Nacional de Padres de Familia y Padres de Alumnos) y la Agrupación de Psicólogos Educativos. Este acuerdo surgió de la necesidad de incorporar y reconocer profesionalmente la figura del psicólogo educativo en los centros educativos, de la necesidad de una mayor profesionalización, con formación, rigor y calidad en las funciones a desarrollar en los centros educativos.

En dicho acuerdo se determinaron las funciones que competen al psicólogo educativo, en las que se incluía la evaluación diagnóstica en contextos educativos, el asesoramiento psicológico a alumnos, padres, profesores y autoridades académicas, la intervención de tipo correctivo, preventivo y optimizador, la coordinación sistemática con otros profesionales y la adecuada derivación en tiempo y forma. También se solicitaba un máster específico y exclusivo para el psicólogo educativo no docente.

Una Comisión Nacional de expertos creada por el Consejo General de Colegios Oficiales de Psicólogos contactó con todos los portavoces de educación de todo el arco parlamentario con el objetivo de sondear la posición de los políticos sobre la posibilidad de regular y legislar sobre el psicólogo educativo no docente. Yo formé parte de esa comisión durante dos años en la que coincidió la última legislatura de Rodríguez Zapatero y la primera legislatura de Mariano Rajoy. La reacción de los políticos fue positiva, redactándose incluso una proposición no de ley en la que figuraba esta posibilidad. La situación finalmente no prosperó debido nuevamente a las divisiones internas de los propios psicólogos ante el posicionamiento de esta nueva salida profesional. Pero sigo considerando que esta especialización es 
necesaria y urgente y resulta perfectamente complementaria con la orientación educativa, pero con la diferencia de que esta salida si es exclusiva para el psicólogo educativo.

Catálogo de actividades profesionales. La primera cuestión planteada en el artículo introductorio es si podemos ponernos de acuerdo en un catálogo de actividades profesionales que definan al psicólogo educativo. Esta labor tendrá que llegar de manera obligada y que se debe abordar necesariamente desde la diversidad de funciones y roles que pueden dar lugar a muchas especialidades. Pero no estoy seguro que lo consigamos desde la psicología educativa española (la división sigue vigente), sino desde la conveniencia y convivencia europea. Ya existe desde hace tiempo la necesidad de establecer criterios de convergencia sobre la formación y campo profesional en Europa. La Federación Europea de Asociaciones de Psicólogos (E.F.P.A.) plantea la necesidad de integrar las funciones y formación especializada del psicólogo educativo en toda la UE, de buscar un marco de referencia europeo para la formación del psicólogo educativo ("EuroPsy"“), un Certificado Europeo de Psicología Educativa que servirá en el futuro como una tarjeta profesional reconocida en toda Europa. Ello se corresponde con la necesidad de que las políticas europeas coincidan en la mejora de la movilidad y en la creación de una calidad de formación común que sea estándar de la profesión. Esta red europea está siendo reforzada por proyectos europeos financiados como el proyecto ESPIL (European School Psychologists improve Lifelong Learning) que ha permitido reunir a representantes de la psicología de los distintos países europeos para varias funciones, entre ellas informar sobre las políticas europeas pertinentes; comparar la situación de los psicólogos educativos de toda Europa; analizar la influencia de las políticas europeas en materia de educación y formación en la profesión; desarrollar una perspectiva sobre cómo los psicólogos pueden contribuir a las políticas europeas relacionadas con el aprendizaje continuo.

Hay, sin embargo, una necesidad de una evaluación realista del sistema educativo: análisis de costes-beneficios. Hay una ausencia de datos accesibles sobre los recursos que se dispone en los países de la UE que permitan establecer conclusiones precisas y contrastadas sobre la eficiencia de estos servicios o recursos. Tampoco existen estudios de análisis de coste-beneficios que evalúen el impacto de los resultados educativos obtenidos. Así, los responsables políticos no disponen de evidencia alguna sobre si los recursos disponibles resultan eficientes, de si se corresponden con otros estándares de calidad desarrollados en otros países o de si estos recursos pueden mejorarse para alcanzar los objetivos deseados. Si la educación es un factor clave en el desarrollo del capital humano, nuestros gobernantes deberían prestan más atención a los resultados de la educación y de la formación propia de cada país, ya se base ésta en análisis cuantitativos o cualitativos, así como en comparaciones internacionales (informe PISA o PIRLS).

Existe también una definición consensuada propuesta por la European Federation of Psychologists' Associations, E.F.P.A. http://www.efpa.eu). La EFPA considera que el profesional que posee una licenciatura en psicología (o grado) y experiencia o formación específica en el campo de la educación y que se engloba bajo el término de "psicólogo educativo". Afecta a todos los psicólogos que trabajan en el ámbito educativo, (centros públicos/privados, escuela infantil, primaria o centros de enseñanza secundaria), rango de alumnos entre

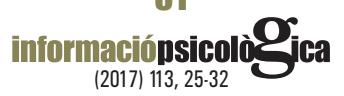




\section{dossier}

los 2 y los 20 años. También incluye a aquellos psicólogos que trabajan en servicios sociales y aprendizaje no formal (e.g., Servicios psicopedagógicos municipales, Servicios de orientación u otros centros o instituciones en los que los psicólogos realizan funciones educativas). Esto podría ser el inicio de un entendimiento futuro. Ojala fuese así.

\section{Referencias}

Escudero, I. y León, J.A.. (2011). Hitos y Retos del Psicólogo Educativo. Psicología Educativa, 17(1), 3-11. http://dx.doi. org/10.5093/ed2011v17n1a1

Méndez Zaballos, Laura. (2011). El Psicólogo Educativo en España. Algunas Propuestas para la Reflexión. Psicología Educativa, 17(1), 39-56. http://dx.doi.org/10.5093/ed2011v17n1a4

León, J.A. (2011). El Psicólogo Educativo en Europa. Psicología Educativa, 17(1), 65-83. http://dx.doi.org/10.5093/ $\underline{\text { ed2011v17n1a6 }}$

Pérez Solís, M. (2011). ¿Por qué un Máster en Psicología Educativa?. Psicología Educativa, 17(1), 101-114.http://dx.doi. org/10.5093/ed2011v17n1a8 